\section{JTI}

JOURNAL OF

TRAUMA AND INJURY

Received: September 14, 2020

Revised: October 24, 2020

Accepted: October 28, 2020

Correspondence to

Seo Young Ko, M.D.

Department of Emergency Medicine, Jeju National University Hospital, 15 Aran

13-gil, Jeju 63241, Korea

Tel: +82-64-717-1904

Fax: +82-64-717-1168

E-mail: koseoyoung84@gmail.com

\title{
Prognostic Accuracy of the Quick Sequen- tial Organ Failure Assessment for Outcomes Among Patients with Trauma in the Emer- gency Department: A Comparison with the Modified Early Warning Score, Revised Trauma Score, and Injury Severity Score
}

\author{
Min Woo Kang, M.D. ${ }^{1}$, Seo Young Ko, M.D. ${ }^{1}$, Sung Wook Song, M.D. ${ }^{1,2}$, \\ Woo Jeong Kim, M.D., Ph.D. ${ }^{1,2}$, Young Joon Kang, M.D., Ph.D. ${ }^{1,3}$, \\ Kyeong Won Kang, M.D., Ph.D., ${ }^{1,2}$, Hyun Soo Park, M.D., Ph.D. ${ }^{1,2}$, \\ Chang Bae Park, M.D. ${ }^{1}$, Jeong Ho Kang, M.D. ${ }^{1,2}$, Ji Hwan Bu, M.D. ${ }^{1}$, \\ Sung Kgun Lee, M.D. ${ }^{1,2}$ \\ ${ }^{1}$ Department of Emergency Medicine, Jeju National University Hospital, Jeju, Korea \\ ${ }^{2}$ Department of Emergency Medicine, Jeju National University School of Medicine, Jeju, Korea \\ ${ }^{3}$ Department of Medical Education, Jeju National University School of Medicine, Jeju, Korea
}

Purpose: To evaluate the severity of trauma, many scoring systems and predictive models have been presented. The quick Sequential Organ Failure Assessment (qSOFA) is a simple scoring system based on vital signs, and we expect it to be easier to apply to trauma patients than other trauma assessment tools.

Methods: This study was a cross-sectional study of trauma patients who visited the emergency department of Jeju National University Hospital. We excluded patients under the age of 18 years and unknown outcomes. We calculated the qSOFA, the Modified Early Warning Score (mEWS), Revised Trauma Score (RTS), and Injury Severity Score (ISS) based on patients' initial vital signs and assessments performed in the emergency department (ED). The primary outcome was mortality within 14 days of trauma. We analyzed qSOFA scores using multivariate logistic regression analysis and compared the predictive accuracy of these scoring systems using the area under the receiver operating characteristic curve (AUROC).

Results: In total, 27,764 patients were analyzed. In the multivariate logistic regression analysis of the qSOFA, the adjusted odds ratios with 95\% confidence interval (CI) for mortality relative to a qSOFA score of 0 were 27.82 (13.63-56.79) for a qSOFA score of 1 , 373.31 (183.47-759.57) for a qSOFA score of 2, and 494.07 (143.75-1698.15) for a qSOFA score of 3. In the receiver operating characteristic (ROC) curve analysis for the qSOFA, mEWS, ISS, and RTS in predicting the outcomes, for mortality, the AUROC for the qSOFA (AUROC [95\% CI]; 0.912 [0.871-0.952]) was significantly greater than those for the ISS (0.700 [0.608-0.793]) and RTS (0.160 [0.108-0.211]).

Conclusions: The qSOFA was useful for predicting the prognosis of trauma patients evaluated in the ED.

Keywords: Trauma severity indices; Sequential Organ Failure Assessment Scores; Mortality; Emergency medicine 


\section{INTRODUCTION}

As a leading cause of death and disability, trauma is one of the most important public health issues. Because trauma has a high incidence in individuals younger than 40 years, the burden derived from trauma, including economic costs, is enormous [1].

This study aimed to evaluate the severity of trauma in patients at the early stage and to provide fast and appropriate treatment in the emergency department (ED). The prognosis of trauma patients depending on their characteristics (such as age) and trauma (such as the site of the injury and degree of sustained damage) [2]. Many scoring systems and predictive models have been developed to evaluate the severity of trauma in patients, such as the Injury Severity Score (ISS), Revised Trauma Score (RTS) and Trauma Revised Injury Severity Score (TRISS) [3-5]. However, no formalized severity assessment method yet exists.

The quick Sequential Organ Failure Assessment (qSOFA) score was developed to assess the prognosis of patients with infectious diseases. It is well known that, similar to trauma patients, fast and appropriate decision-making helps the treatment of patients with sepsis [6]. Finding a simple way to assess a patient's condition using limited information in the ED, including vital signs, is of paramount importance in providing patients with quick and appropriate treatment, and the simpler the information, the better [7]. The qSOFA consists of three indicators (the Glasgow Coma Scale [GCS], systolic blood pressure $[\mathrm{SBP}]$, and respiratory rate $[\mathrm{RR}])$, similar to the RTS, and uses a simpler formula than the RTS. Therefore, we expected to be able to use the qSOFA scoring system quickly and easily in prehospital care and the ED to evaluate trauma patients. In a recent study, the qSOFA score was used to evaluate patients with infectious and non-infectious diseases in the ED and found to be useful [8]. Another study showed that higher qSOFA scores were associated with greater injury severity, increased intensive care unit (ICU) admission, and in-hospital mortality in patients with blunt trauma [9].

The qSOFA is evaluated based on only three diagnostic criteria and can be applied to trauma patients more easily than previous trauma assessment tools. This study ana- lyzed the associations between the qSOFA and outcomes of trauma patients in the ED. We attempted to validate the qSOFA scoring system for evaluating the severity of trauma patients relative to other scoring systems.

\section{METHODS}

\section{Study design and setting}

This study was a retrospective cross-sectional study of trauma patients who visited the ED of Jeju National University Hospital from January 1, 2013 to November 30, 2017. The Jeju National University Hospital is part of a nationwide multicenter prospective registry study, the Emergency Department-based Injury Surveillance System (EDISS). This registry study was conducted to investigate the epidemiology of injuries at 23 EDs in tertiary hospitals operated by the Korea Centers for Disease Prevention and Control.

\section{Study population}

The eligible population included all patients who visited the ED at Jeju National University Hospital due to trauma between January 1, 2013 and November 30, 2017. The study population was adults who were 18 years of age or older on the day of the incident. Patients without information on outcomes, those who had died upon arrival at the scene, and those who were transferred to another hospital were excluded. Patients who had insufficient information available to calculate the qSOFA, modified Early Warning Score (mEWS), RTS, and ISS were excluded from the final analysis.

\section{Data collection}

We analyzed data drawn from the EDISS database. These prospective multicenter registry data were collected by ED physicians at 23 participating hospitals. The registry included the following comprehensive information for trauma: patient demographics (age, sex, insurance type, past medical history, vital signs and mental status), injury characteristics (intention, mechanism, activities, places, body site of injury, emergency medical service usage, and whether the injury was alcohol-related), injury severity, emergency care process, diagnosis, treatment, outcome 
(discharge, inter-hospital transfer, admission, death, and others) at the ED, and outcome after hospital admission (discharge, inter-hospital transfer, death, and other). The missing data were collected directly through electronic medical record review by the researchers.

\section{Variables and outcomes}

The variables included age, sex, alcohol use, insurance, use of emergency medical services (EMS), whether the injury occurred on the weekend, the hour of the event, the patient's past medical history (patient-related factors), intention, mechanism of injury, place of event, activity at the time of injury, and the site of the injury (injury-related factors).

We calculated the qSOFA, mEWS, RTS, and ISS from the patient's initial vital signs and assessment at the ED. The qSOFA variables included the GCS, SBP, and RR. Each variable that met the criteria (GCS $\leq 14, \mathrm{SBP} \leq 100$ $\mathrm{mmHg}$, and $\mathrm{RR} \geq 22$ breaths per minute) was given 1 point, otherwise a score of 0 points was assigned. The qSOFA scores ranged from 0 to 3 , with a higher score indicating more severe disease [10-12]. The mEWS was calculated from vital signs (RR, heart rate, SBP, and body temperature) and mental status (AVPU) $[13,14]$. The RTS was calculated by applying a special expression including weights using the same variables as the qSOFA. The ISS was calculated according to the evaluation of the patient's injury site and injury severity [15].

The primary outcome was mortality within 14 days of trauma. The secondary outcomes were emergency surgery performed within 3 days of trauma and ICU admission from the ED.

\section{Statistical analysis}

Demographic findings were compared for the qSOFA score. Statistical tests were performed, including the chisquare test, analysis of variance, and the Kruskal-Wallis test for categorical variables depending on the distribution of the data. Descriptive statistics are presented as frequencies and percentages for categorical variables. We calculated the means, medians, and interquartile ranges for continuous variables depending on their distribution.

The adjusted odds ratios (aORs) with 95\% confidence intervals (95\% CIs) of the study outcomes were calculated for qSOFA scores using multivariate logistic regression analysis, adjusted for potential confounders. We also conducted additional multiple comparisons using a linear prediction model. Using a cutoff for the qSOFA score, we calculated the sensitivity, specificity, positive likelihood ratio, and negative likelihood ratio. We compared the predictive accuracy of the various scoring systems, using the area under the receiver operating characteristic (AUROC) curve and 95\% CIs. All statistical analyses were performed using Stata version 14.0 (Stata Corp, College Station, TX, USA), utilizing two-tailed tests with statistical significance defined as $p<0.05$.

\section{Ethics statements}

The study complied with the Declaration of Helsinki, and its protocol was approved by the Jeju National University Hospital Institutional Review Board (IRB) with a waiver for the need for informed consent (IRB No. JEJUNUH 2020-04-001).

\section{RESULTS}

A total of 39,534 trauma patients visited the ED during the study period and 27,764 patients were finally analyzed. We excluded patients younger than 18 years $(n=11,721)$ and those with unknown outcomes ( $\mathrm{n}=49$ ) (Fig. 1).

In total, $94.8 \%$ of patients had a qSOFA score of $0,4.5 \%$ had a qSOFA score of $1,0.6 \%$ had a qSOFA score of 2 , and $0.1 \%$ had a qSOFA score of 3 . Most demographic findings were significantly different according to qSOFA scores. Table 1 shows the patient-related factors by qSO-

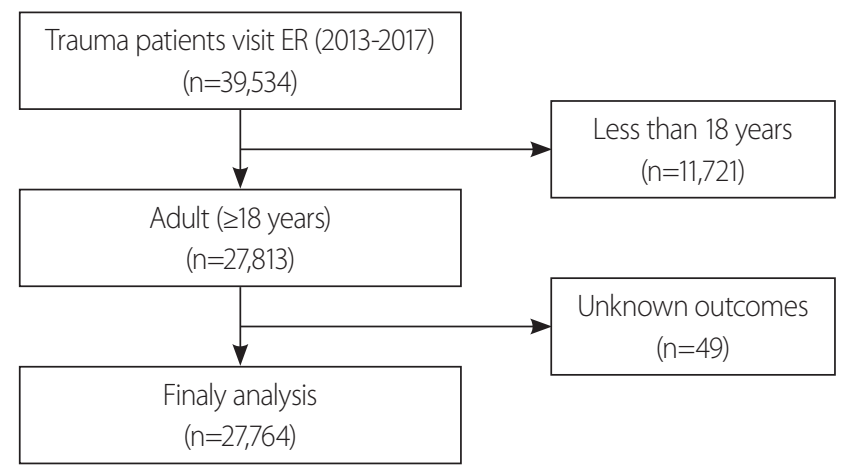

Fig. 1. Study population. ER: emergency room. 
Table 1. Demographics characteristics of patient-related factors by qSOFA scores

\begin{tabular}{|c|c|c|c|c|c|c|}
\hline qSOFA & Total & 0 & 1 & 2 & 3 & $p$-value \\
\hline Total & $27,764(100.0)$ & $26,317(100.0)$ & 1,252 (100.0) & 175 (100.0) & $20(100.0)$ & \\
\hline Age & & & & & & $<0.001$ \\
\hline Mean (q25-q75) & $46.7(32.0-59.0)$ & $46.5(31.0-59.0)$ & $50.7(35.0-67.0)$ & $53.2(39.0-65.0)$ & $55.1(44.5-67.5)$ & \\
\hline Gender & & & & & & 0.152 \\
\hline Male & $15,660(56.4)$ & $14,848(56.4)$ & $690(55.1)$ & $112(64.0)$ & $10(50.0)$ & \\
\hline Female & $12,104(43.6)$ & $11,469(43.6)$ & $562(44.9)$ & $63(36.0)$ & $10(50.0)$ & \\
\hline Alcohol use & & & & & & $<0.001$ \\
\hline Yes & 2,557 (9.2) & 2,334 (8.9) & $178(14.2)$ & $40(22.9)$ & $5(25.0)$ & \\
\hline No & $21,724(78.2)$ & 20,727 (78.8) & 897 (71.6) & $93(53.1)$ & $7(35.0)$ & \\
\hline Unknown & $3,462(12.5)$ & $3,242(12.3)$ & 170 (13.6) & $42(24.0)$ & $8(40.0)$ & \\
\hline Missing & $21(0.1)$ & $14(0.1)$ & $7(0.6)$ & $0(0.0)$ & $0(0.0)$ & \\
\hline Insurance & & & & & & $<0.001$ \\
\hline $\mathrm{NHI}$ & $19,826(71.4)$ & 18,887 (71.8) & $824(65.8)$ & $105(60.0)$ & $10(50.0)$ & \\
\hline Medical aid & $1,352(4.9)$ & $1,238(4.7)$ & $97(7.7)$ & $13(7.4)$ & $4(20.0)$ & \\
\hline Car insurance & $5,988(21.6)$ & $5,631(21.4)$ & $298(23.8)$ & $53(30.3)$ & $6(30.0)$ & \\
\hline Others & $470(1.7)$ & $435(1.7)$ & $32(2.6)$ & $3(1.7)$ & $0(0.0)$ & \\
\hline Missing & $128(0.5)$ & $126(0.5)$ & $1(0.1)$ & $1(0.6)$ & $0(0.0)$ & \\
\hline EMS use & & & & & & $<0.001$ \\
\hline EMS ambulance & 7,967 (28.7) & $7,219(27.4)$ & $605(48.3)$ & $128(73.1)$ & 15 (75.0) & \\
\hline Other ambulance & 615 (2.2) & $510(1.9)$ & $77(6.2)$ & $23(13.1)$ & $5(25.0)$ & \\
\hline Walk \& car & $18,920(68.1)$ & $18,351(69.7)$ & $548(43.8)$ & $21(12.0)$ & $0(0.0)$ & \\
\hline Unknown & $230(0.8)$ & $210(0.8)$ & $17(1.4)$ & $3(1.7)$ & $0(0.0)$ & \\
\hline Yes & $7,967(28.7)$ & $7,219(27.4)$ & $605(48.3)$ & $128(73.1)$ & $15(75.0)$ & \\
\hline No & $19,797(71.3)$ & $19,098(72.6)$ & $647(51.7)$ & $47(26.9)$ & $5(25.0)$ & \\
\hline Week & & & & & & $<0.001$ \\
\hline Weekday & $18,517(66.7)$ & $17,453(66.3)$ & $923(73.7)$ & $126(72.0)$ & $15(75.0)$ & \\
\hline Weekend & $9,247(33.3)$ & $8,864(33.7)$ & $329(26.3)$ & $49(28.0)$ & $5(25.0)$ & \\
\hline Hour & & & & & & $<0.001$ \\
\hline Day (9-18 hours) & $15,066(54.3)$ & $14,263(54.2)$ & $719(57.4)$ & $78(44.6)$ & $6(30.0)$ & \\
\hline Night & $12,698(45.7)$ & $12,054(45.8)$ & $533(42.6)$ & $97(55.4)$ & $14(70.0)$ & \\
\hline \multicolumn{7}{|l|}{ Past medical history } \\
\hline Hypertension & $2,826(10.2)$ & $2,622(10.0)$ & $167(13.3)$ & $33(18.9)$ & $4(20.0)$ & $<0.001$ \\
\hline Diabetes & $1,319(4.8)$ & $1,209(4.6)$ & $90(7.2)$ & $16(9.1)$ & $4(20.0)$ & $<0.001$ \\
\hline Tuberculosis & $33(0.1)$ & $31(0.1)$ & $1(0.1)$ & $1(0.6)$ & $0(0.0)$ & 0.361 \\
\hline Liver disease & $265(1.0)$ & $240(0.9)$ & $22(1.8)$ & $2(1.1)$ & $1(5.0)$ & 0.006 \\
\hline Stroke & $587(2.1)$ & $537(2.0)$ & $47(3.8)$ & $3(1.7)$ & $0(0.0)$ & 0.001 \\
\hline Heart disease & $667(2.4)$ & $614(2.3)$ & $42(3.4)$ & $10(5.7)$ & $1(5.0)$ & 0.003 \\
\hline Cancer & $357(1.3)$ & $321(1.2)$ & $32(2.6)$ & $4(2.3)$ & $0(0.0)$ & $<0.001$ \\
\hline
\end{tabular}

Values are presented as number (\%) or median (interquartile range).

qSOFA: quick Sepsis-related Organ Failure Assessment, NHI: national health insurance, EMS: emergency medical service. 
Table 2. Characteristics of injury-related factors by qSOFA scores

\begin{tabular}{|c|c|c|c|c|c|c|}
\hline qSOFA & Total & 0 & 1 & 2 & 3 & $p$-value \\
\hline Total & $27,764(100.0)$ & $26,317(100.0)$ & $1,252(100.0)$ & $175(100.0)$ & $20(100.0)$ & \\
\hline Intention & & & & & & $<0.001$ \\
\hline Accidental & $25,625(92.3)$ & $24,426(92.8)$ & 1,075 (85.9) & $110(62.9)$ & $14(70.0)$ & \\
\hline Self-harm/suicide & $537(1.9)$ & $380(1.4)$ & $99(7.9)$ & $53(30.3)$ & $5(25.0)$ & \\
\hline Assault & $1,402(5.0)$ & $1,352(5.1)$ & $45(3.6)$ & $4(2.3)$ & $1(5.0)$ & \\
\hline Unknown & $170(0.6)$ & $134(0.5)$ & $28(2.2)$ & $8(4.6)$ & $0(0.0)$ & \\
\hline Mechanism of injury & & & & & & $<0.001$ \\
\hline Fall & $7,424(26.7)$ & $7,027(26.7)$ & $364(29.1)$ & $28(16.0)$ & $5(25.0)$ & \\
\hline Motor vehicle crash & $7,158(25.8)$ & $6,713(25.5)$ & $368(29.4)$ & $70(40.0)$ & $7(35.0)$ & \\
\hline Blunt & $4,651(16.8)$ & $4,496(17.1)$ & 145 (11.6) & $8(4.6)$ & $2(10.0)$ & \\
\hline Penetrating & 3,210 (11.6) & 3,105 (11.8) & $99(7.9)$ & $5(2.9)$ & $1(5.0)$ & \\
\hline Others & $5,321(19.2)$ & $4,976(18.9)$ & $276(22.0)$ & 64 (36.6) & $5(25.0)$ & \\
\hline Place & & & & & & $<0.001$ \\
\hline Residence & $7,338(26.4)$ & $6,867(26.1)$ & 402 (32.1) & $60(34.3)$ & $9(45.0)$ & \\
\hline Road & $9,015(32.5)$ & $8,496(32.3)$ & $438(35.0)$ & $74(42.3)$ & $7(35.0)$ & \\
\hline Commercial place & $2,437(8.8)$ & 2,344 (8.9) & $84(6.7)$ & $7(4.0)$ & $2(10.0)$ & \\
\hline Hospital & $323(1.2)$ & $301(1.1)$ & $21(1.7)$ & $1(0.6)$ & $0(0.0)$ & \\
\hline Work place & $3,123(11.2)$ & 2,983 (11.3) & $126(10.1)$ & $13(7.4)$ & $1(0.0)$ & \\
\hline Unspecified & $5,528(19.9)$ & $5,326(20.2)$ & $181(14.5)$ & $20(11.4)$ & $1(5.0)$ & \\
\hline Activity at injury & & & & & & $<0.001$ \\
\hline Paid work & $5,398(19.4)$ & $5,164(19.6)$ & $202(16.1)$ & $30(17.1)$ & $2(10.0)$ & \\
\hline Unpaid work & $8,260(29.8)$ & $7,844(29.8)$ & $376(30.0)$ & $35(20.0)$ & $5(25.0)$ & \\
\hline Vital activity & 8,898 (32.0) & $8,447(32.1)$ & $401(32.0)$ & $43(24.6)$ & $7(35.0)$ & \\
\hline Leisure & $1,634(5.9)$ & $1,568(6.0)$ & $62(5.0)$ & $3(1.7)$ & $1(5.0)$ & \\
\hline Others & $3,574(12.9)$ & $3,294(12.5)$ & $211(16.9)$ & $64(36.6)$ & $5(25.0)$ & \\
\hline \multicolumn{7}{|l|}{ Body site of injury } \\
\hline Head & $9,039(32.6)$ & $8,495(32.3)$ & 457 (36.5) & 75 (42.9) & $12(60.0)$ & $<0.001$ \\
\hline Neck & $3,381(12.2)$ & $3,245(12.3)$ & $123(9.8)$ & $12(6.9)$ & $1(5.0)$ & 0.005 \\
\hline Thorax & $2,229(8.0)$ & $1,921(7.3)$ & $247(19.7)$ & $50(28.6)$ & $11(55.0)$ & $<0.001$ \\
\hline Adomen/pelvis & 3,301 (11.9) & 3,039 (11.5) & $201(16.1)$ & $51(29.1)$ & $10(50.0)$ & $<0.001$ \\
\hline Upper extremity & $1,868(6.7)$ & $1,743(6.6)$ & $107(8.5)$ & $17(9.7)$ & $1(5.0)$ & 0.022 \\
\hline Elbow & 2,117 (7.6) & 1,991 (7.6) & $112(8.9)$ & $13(7.4)$ & $1(5.0)$ & 0.329 \\
\hline Wrist/hand & $4,161(15.0)$ & $4,002(15.2)$ & 145 (11.6) & $13(7.4)$ & $1(5.0)$ & $<0.001$ \\
\hline Lower extremity & $1,576(5.7)$ & $1,463(5.6)$ & $96(7.7)$ & $13(7.4)$ & $4(20.0)$ & $<0.001$ \\
\hline Knee & $3,560(12.8)$ & $3,364(12.8)$ & 165 (13.2) & $27(15.4)$ & $4(20.0)$ & 0.539 \\
\hline Foot & 2,181 (7.9) & 2,115 (8.0) & $60(4.8)$ & $5(2.9)$ & $1(5.0)$ & $<0.001$ \\
\hline
\end{tabular}

Values are presented as number (\%).

qSOFA: quick Sepsis-related Organ Failure Assessment. 
Table 3. Outcomes based on qSOFA scores

\begin{tabular}{|c|c|c|c|c|c|}
\hline qSOFA & Total & 0 & 1 & 2 & 3 \\
\hline Total (\%) & $27,764(100.0)$ & $26,317(100.0)$ & $1,252(100.0)$ & $175(100.0)$ & $20(100.0)$ \\
\hline \multicolumn{6}{|l|}{ ICU admission } \\
\hline N (\%) & $649(2.3)$ & $343(1.3)$ & $203(16.2)$ & $88(50.3)$ & $15(75.0)$ \\
\hline OR $(95 \% \mathrm{Cl})$ & & Reference & 14.65 (12.19-17.62) & 76.6 (55.91-104.95) & $227.18(82.11-628.57)$ \\
\hline $\mathrm{aOR} \mathrm{R}^{\mathrm{a}}(95 \% \mathrm{Cl})$ & & Reference & $11.46(9.46-13.89)$ & $46.84(33.56-65.37)$ & $142.89(50.10-407.47)$ \\
\hline \multicolumn{6}{|c|}{ Emergency surgery } \\
\hline $\mathrm{N}(\%)$ & $1,269(4.6)$ & $1,151(4.4)$ & $92(7.3)$ & $22(12.6)$ & $4(20.0)$ \\
\hline OR $(95 \% \mathrm{Cl})$ & & Reference & $1.73(1.39-2.16)$ & $3.14(2.00-4.93)$ & $5.47(1.82-16.38)$ \\
\hline $\mathrm{aOR}{ }^{\mathrm{a}}(95 \% \mathrm{Cl})$ & & Reference & $1.56(1.24-1.95)$ & $2.69(1.69-4.27)$ & $5.55(1.81-17.04)$ \\
\hline \multicolumn{6}{|l|}{ Mortality } \\
\hline N (\%) & $80(0.3)$ & $12(0.0)$ & $23(1.8)$ & $29(16.6)$ & $6(30.0)$ \\
\hline $\mathrm{OR}(95 \% \mathrm{Cl})$ & & Reference & $41.02(20.37-82.64)$ & $628.61(322.12-1,226.73)$ & $939.46(309.22-2,854.27)$ \\
\hline $\mathrm{aOR}{ }^{\mathrm{a}}(95 \% \mathrm{Cl})$ & & Reference & $27.82(13.63-56.79)$ & 373.31 (183.47-759.57) & $494.07(143.75-1,698.15)$ \\
\hline
\end{tabular}

qSOFA: quick Sepsis-related Organ Failure Assessment, ICU: intensive care unit, OR: odds ratio, Cl: confidence interval.

adjusted: gender, age, alcohol, insurance, use of emergency medical services, intention, past medical history (hypertension, diabetes, tuberculosis, liver disease, stroke, heart disease, cancer), weekday, hour.

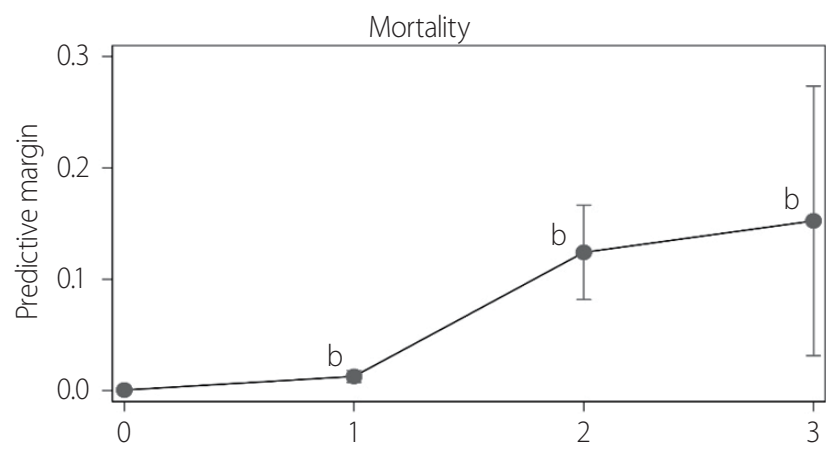

A

qSOFA score

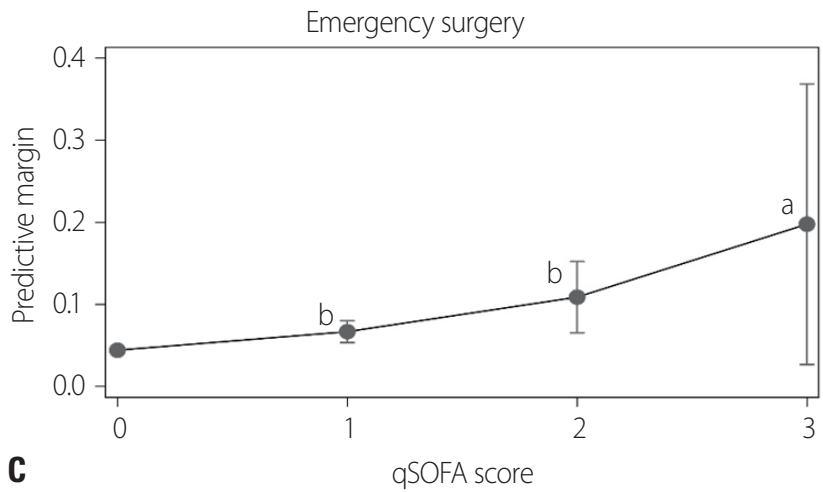

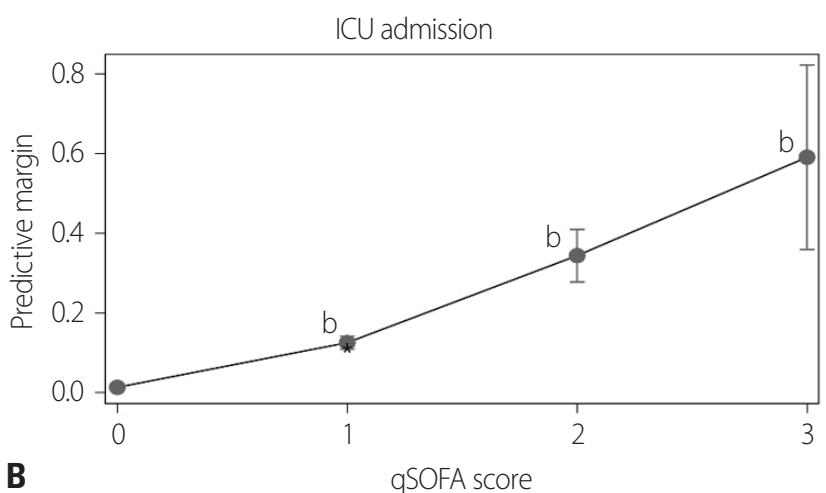

Fig. 2. Adjusted predictive margins of overall outcomes. Adjusted predictive margins of (A) mortality in 14 days, (B) ICU admission, (C) emergency surgery in 3 days. This figure shows the results obtained from multivariable logistic regression for outcomes followed by additional multiple comparisons of the qSOFA score. Reference qSOFA=0. qSOFA: quick Sequential Organ Failure Assessment, ICU: intensive care unit. ${ }^{a} p$ for trend $<0.05$. ${ }^{b} p$ for trend $<0.001$. 
FA scores, and Table 2 shows the injury-related factors according to qSOFA scores.

From the multivariate logistic regression analysis for qSOFA, the aOR $(95 \% \mathrm{CI})$ for mortality relative to a qSOFA score of 0 was 27.82 (13.63-56.79) for a qSOFA score of $1,373.31$ (183.47-759.57) for a qSOFA score of 2, and 494.07 (143.75-1698.15) for a qSOFA score of 3 in the adjusted model. The aOR ( $95 \% \mathrm{CI})$ for emergency surgery relative to a qSOFA score of 0 was $1.56(1.39-2.16)$ for a qSOFA score of 1, 2.69 (1.69-4.27) for a qSOFA score of 2 , and 5.55 (1.81-17.04) for a qSOFA score of 3 . The aOR (95\% CI) for admission to the ICU relative to a qSOFA score of 0 was $11.46(9.46-13.89)$ for a qSOFA score of 1 , $46.84(33.56-65.37)$ for a qSOFA score of 2 , and 142.89
(50.10-407.47) for a qSOFA score of 3 in the adjusted model (Table 3). Fig. 2 shows the results obtained from the multivariate logistic regression for overall outcomes followed by additional multiple comparisons of the qSOFA. There was a statistically significant increase in the outcomes at qSOFA scores of 1,2 , and 3 relative to reference (a qSOFA score of 0 ).

Table 4 shows the sensitivity, specificity, positive likelihood ratio, and negative likelihood ratio according to the qSOFA cutoff value. Figs. 3, 4 and Table 5 show the receiver operating characteristic curves for the qSOFA, mEWS, ISS, and RTS for predicting the outcomes. For mortality, the AUROC for the qSOFA (AUROC [95\% $\mathrm{CI}] ; 0.912$ [0.871-0.952]) was significantly greater than

Table 4. Test characteristics of qSOFA scores for outcomes in trauma patients

\begin{tabular}{|c|c|c|c|c|}
\hline Cut-off value of qSOFA & Sensitivity (\%) & Specificity (\%) & $\operatorname{LR}(+)$ & $\operatorname{LR}(-)$ \\
\hline \multicolumn{5}{|l|}{ Mortality } \\
\hline$\geq 1$ & 85.0 & 95.0 & 17.06 & 0.16 \\
\hline$\geq 2$ & 56.3 & 99.5 & 103.81 & 0.44 \\
\hline$\geq 3$ & 7.5 & 100.0 & 148.32 & 0.93 \\
\hline \multicolumn{5}{|c|}{ ICU admission and emergency surgery } \\
\hline$\geq 1$ & 20.4 & 95.9 & 4.93 & 0.83 \\
\hline$\geq 2$ & 6.0 & 99.7 & 18.11 & 0.94 \\
\hline$\geq 3$ & 0.8 & 100.0 & 42.86 & 0.99 \\
\hline
\end{tabular}

LR: likelyhood ratio, ICU: intensive care unit.

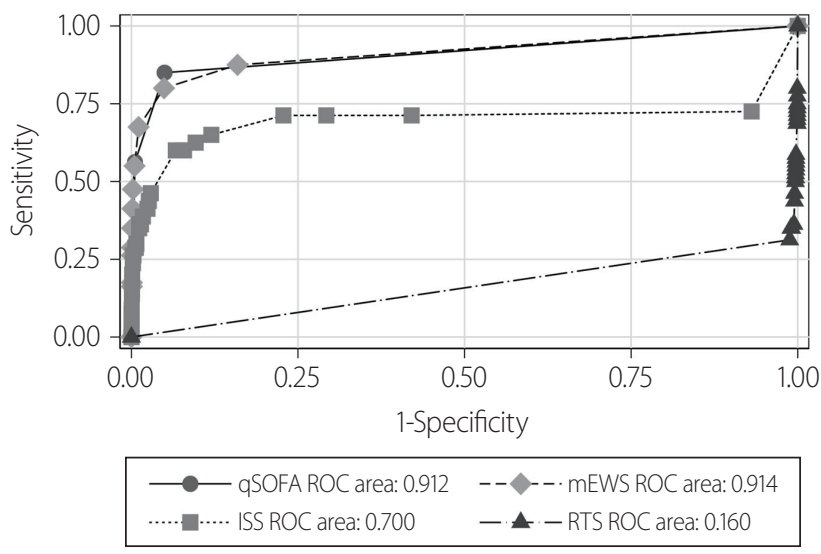

Fig. 3. The receiver operating characteristic curve for in-hospital mortality relative to the predictive power of various scoring systems (qSOFA, mEWS, RTS, and ISS). qSOFA: quick Sequential Organ Failure Assessment, mEWS: modified Early Warning Score, RTS: Revised Trauma Score, ISS: Injury Severity Score.

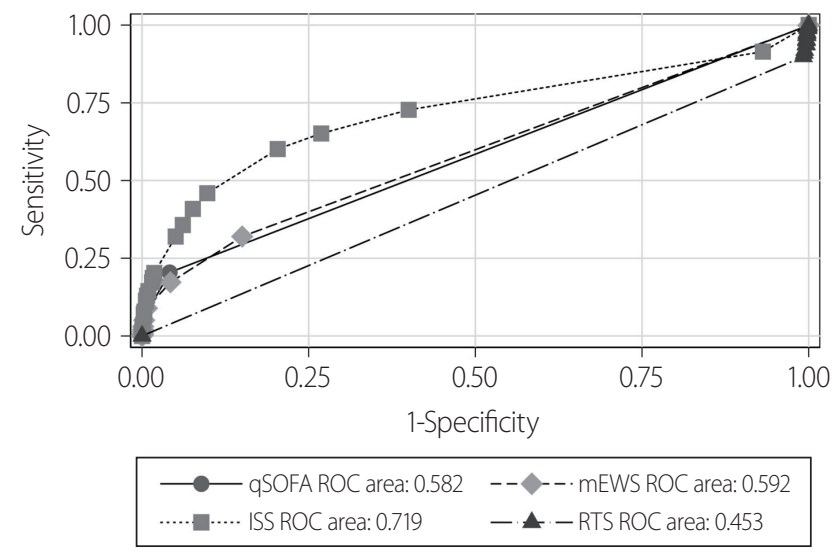

Fig. 4. The receiver operating characteristic curves for ICU admission and emergency surgery relative to the predictive power of various scoring systems (qSOFA, mEWS, RTS, and ISS). qSOFA: quick Sequential Organ Failure Assessment, mEWS: Modified Early Warning Score, RTS: Revised Trauma Score, ISS: Injury Severity Score, ICU: intensive care unit. 
those for the ISS $(0.700$ [0.608-0.793]) and RTS $(0.160$ [0.108-0.211]). The AUROC for the mEWS (0.914 [0.873-0.956]) was similar to that for qSOFA (0.914 [0.873-0.956]) (Table 5, Fig. 3). For ICU admission and emergency surgery, the AUROC for the qSOFA (AUROC [95\% CI]; 0.582 [0.573-0.592]) was significantly greater than that for the RTS $(0.453[0.446-0.460])$ and smaller those that for the ISS $(0.719$ [0.704-0.734] $)$ and mEWS (0.592 [0.580-0.603]) (Table 5, Fig. 4).

\section{DISCUSSION}

Trauma patients with a higher qSOFA score in the ED had higher mortality and higher chances of both ICU admission and emergency surgery. The outcome measures for patients with qSOFA scores of 1, 2, and 3 were significantly higher relative to the reference (a qSOFA score of 0 ). We compared the predictive accuracy of various scoring systems (qSOFA, mEWS, ISS, and RTS) using AUROC curves. For mortality, the AUROC of the qSOFA was significantly greater than that of the ISS and RTS. There was no significant difference between the AUROCs of the qSOFA and the mEWS. Since the qSOFA uses only three factors (GCS, SBP and RR), and uses "yes/no" answers, it is easier to utilize for evaluating trauma patient than the mEWS at the moment of patient arrival to the ED. This is an advantage of the qSOFA.
Our study showed that qSOFA scores were statistically significantly correlated with outcomes (mortality within 14 days, ICU admission, and emergency surgery within 3 days) of trauma patients visiting the ED. The predictive power of the qSOFA for in-hospital mortality was similar or superior to that of the mEWS, RTS and ISS in this study, but qSOFA scores can be calculated more simply and quickly than the mEWS, RTS, and ISS. We analyzed all trauma patients who presented to the ED, so our study was highly representative of this population. Our study also showed that using the qSOFA to predict mortality was superior in the target group relative to other scoring systems such as the RTS and ISS. These are the main strengths of our study. The 2016 SCCM/ESICM guideline proposed the concept of the qSOFA score to predict poor outcomes in patients with suspected infections [11]. Several recent studies have shown that qSOFA scores were also useful in assessing outcomes in patients with noninfectious diseases $[8,9,14,16]$. Similar to our study, these studies have shown that the qSOFA may play a role in the prediction of mortality during the evaluation of trauma patients. Jawa et al. [9] investigated patients with blunt trauma who were hospitalized from the ED, and found that qSOFA scores showed similar associations with mortality, ICU hospitalization, and major surgery to the RTS and TRISS (the AUROC of qSOFA scores was 0.73 for inhospital mortality, 0.66 for ICU admission, and 0.53 for major surgery). This study excluded patients who died in

Table 5. Comparison of various scoring systems for outcomes using the AUROC

\begin{tabular}{|c|c|c|c|c|}
\hline & AUROC & & & $\operatorname{Pr}>\mathrm{chi}^{2}$ \\
\hline \multicolumn{5}{|l|}{ Mortality } \\
\hline qSOFA & 0.912 & 0.871 & 0.952 & Reference \\
\hline mEWS & 0.914 & 0.873 & 0.956 & 0.782 \\
\hline ISS & 0.700 & 0.608 & 0.793 & $<0.001$ \\
\hline RTS & 0.160 & 0.108 & 0.211 & $<0.001$ \\
\hline \multicolumn{5}{|c|}{ ICU admission and emergency surgery } \\
\hline qSOFA & 0.582 & 0.573 & 0.592 & Reference \\
\hline mEWS & 0.592 & 0.580 & 0.603 & 0.033 \\
\hline ISS & 0.719 & 0.704 & 0.734 & $<0.001$ \\
\hline RTS & 0.453 & 0.446 & 0.460 & $<0.001$ \\
\hline
\end{tabular}

AUROC: area under the receiver operating characteristic curve, qSOFA: quick Sepsis-related Organ Failure Assessment, mEWS: modified Early Warning Score, ISS: Injury Severity Score, RTS: revised trauma score, ICU: intensive care unit. 
the ED or were not hospitalized from the ED [9]. Miyamoto et al. [12] analyzed trauma patients and showed that the pre-hospital qSOFA score, recorded before arrival at the hospital, was closely related to in-hospital mortality (AUROC $=0.70)$ and showed a significant association with patient mortality within 2 days (AUROC $=0.74$ ). In another study of trauma patients who presented at the ED through an EMS ambulance, it was very interesting to note that the relationship between changes in the qSOFA score and mortality was significant [17]. Kitahara et al. [14] showed that the pre-hospital qSOFA for non-infectious patients had a higher predictive power for in-hospital mortality than the mEWS (AUROC of qSOFA vs. mEWS, 0.70 vs. 0.62 ).

The ISS and RTS are useful scoring systems for the assessing the severity of injury in trauma patients. However, these scores are difficult to use immediately in the ED [15,18-20]. First, to calculate the ISS, we need anatomical information reflective of the severity of the full range of trauma after the final diagnosis. Therefore, the ISS is difficult to use for a rapid initial evaluation of trauma patients in the ED $[4,5]$. The RTS is obtained using three factors (GCS, SBP, RR) that are also used in the qSOFA. Each factor is scored on a five-point scale and each factor is weighted. The RTS is then calculated using a complex formula of the three factors [21]. The mEWS was developed to guide medical providers to quickly determine the degree of illness of a patient in a hospital. It is based on vital signs (AVPU, RR, SBP, pulse/heart rate, and body temperature). The mEWS was designed to help ward staff identify when to call for specialist advice for critical care services in hospitals. Recently, it has been used to evaluate patients in the ED and out-of-hospital $[13,14,22]$. The ISS is useful when comparing the severity of trauma after the final diagnosis, but it is not suitable for predicting the outcomes of trauma patients in the early stages of ED treatment. The RTS is a scoring system that utilizes the same elements as the qSOFA, but the disadvantage of RTS is the complex series of steps used to obtain the score. The qSOFA can be obtained relatively simply in the initial assessment in the ED. We observed that the qSOFA score had greater predictive power than either the ISS or RTS for mortality in this study. Therefore, we believe that the qSOFA can be used as a convenient tool to predict the prognosis of trauma patients in the ED.

This study has several limitations. First, this study was retrospective in nature and analyzed data from a single hospital registry. The second limitation is the relatively low number of patients with a qSOFA score of 3. Finally, we used the initial vital signs and information of patients when they presented to the ED. Consequently, we might have missed patients who deteriorated within a few minutes of arrival at the ED. The qSOFA score could then have been underestimated.

\section{CONCLUSION}

Trauma patients with a higher qSOFA score had higher mortality and a higher chance of ICU admission and emergency surgery. This study shows that using the qSOFA for trauma patient evaluation was useful for predicting patients' prognosis in the ED.

\section{ACKNOWLEDGEMENTS}

This research was supported by Research of Korea Centers for Disease Control and Prevention.

\section{REFERENCES}

1. World Health Organization (WHO). Injuries and violence: the facts [Internet]. Geneva: WHO 2014 [cited 2020 Sep 14]. Available from: https://www.who.int/violence_injury_prevention/ media/news/2015/Injury_violence_facts_2014/en/.

2. Champion HR, Sacco WJ, Copes WS, Gann DS, Gennarelli TA, Flanagan ME. A revision of the trauma score. J Trauma 1989;29:623-9.

3. Kondo Y, Abe T, Kohshi K, Tokuda Y, Cook EF, Kukita I. Revised trauma scoring system to predict in-hospital mortality in the emergency department: Glasgow Coma Scale, age, and systolic blood pressure score. Crit Care 2011;15:R191.

4. Kim YH, Seo KS, Lee MJ, Park JB, Kim JK, Ahn JY, et al. Application of new trauma scoring systems for mortality prediction in patients with adult major trauma. J Korean Soc Emerg Med 2014;25:447-55. 
5. Hwang JY, Lee KH, Shin HJ, Cha KC, Kim H, Hwang SO. Correlation analysis of trauma scoring system in predictive validity in motor vehicle accident. J Korean Soc Emerg Med 2011;22:329-34.

6. Bhattacharjee P, Edelson DP, Churpek MM. Identifying patients with sepsis on the hospital wards. Chest 2017;151:898-907.

7. Freund Y, Lemachatti N, Krastinova E, Van Laer M, Claessens YE, Avondo A, et al. Prognostic accuracy of sepsis-3 criteria for in-hospital mortality among patients with suspected infection presenting to the emergency department. JAMA 2017;317:3018.

8. Singer AJ, Ng J, Thode HC Jr, Spiegel R, Weingart S. Quick SOFA scores predict mortality in adult emergency department patients with and without suspected infection. Ann Emerg Med 2017;69:475-9.

9. Jawa RS, Vosswinkel JA, McCormack JE, Huang EC, Thode HC Jr, Shapiro MJ, et al. Risk assessment of the blunt trauma victim: the role of the quick Sequential Organ Failure Assessment score (qSOFA). Am J Surg 2017;214:397-401.

10. Seymour CW, Liu VX, Iwashyna TJ, Brunkhorst FM, Rea TD, Scherag A, et al. Assessment of clinical criteria for sepsis: for the third international consensus definitions for sepsis and septic shock (sepsis-3). JAMA 2016;315:762-74.

11. Singer M, Deutschman CS, Seymour CW, Shankar-Hari M, Annane D, Bauer M, et al. The third international consensus definitions for sepsis and septic shock (sepsis-3). JAMA 2016;315:801-10.

12. Miyamoto K, Shibata N, Ogawa A, Nakashima T, Kato S. Prehospital quick Sequential Organ Failure Assessment score to predict in-hospital mortality among patients with trauma. Am J Emerg Med 2019;37:2165-70.

13. Subbe CP, Kruger M, Rutherford P, Gemmel L. Validation of a modified early warning score in medical admissions. QJM 2001;94:521-6.
14. Kitahara O, Nishiyama K, Yamamoto B, Inoue S, Inokuchi S. The prehospital quick SOFA score is associated with in-hospital mortality in noninfected patients: a retrospective, cross-sectional study. PLoS One 2018;13:e0202111.

15. Boyd CR, Tolson MA, Copes WS. Evaluating trauma care: the TRISS method. Trauma score and the Injury Severity Score. J Trauma 1987;27:370-8.

16. LeGuen M, Ballueer Y, McKay R, Eastwood G, Bellomo R, Jones D; Austin Health RRT qSOFA investigators. Frequency and significance of qSOFA criteria during adult rapid response team reviews: a prospective cohort study. Resuscitation 2018;122:1318.

17. Miyamoto K, Shibata N, Ogawa A, Nakashima T, Kato S. Prehospital and in-hospital quick Sequential Organ Failure Assessment (qSOFA) scores to predict in-hospital mortality among trauma patients: an analysis of nationwide registry data. Acute Med Surg 2020;7:e532.

18. Baker SP, O’Neill B, Haddon W Jr, Long WB. The Injury Severity Score: a method for describing patients with multiple injuries and evaluating emergency care. J Trauma 1974;14:187-96.

19. Champion HR, Sacco WJ, Carnazzo AJ, Copes W, Fouty WJ. Trauma score. Crit Care Med 1981;9:672-6.

20. Jung K, Lee JCJ, Kim J. Injury Severity Scoring system for trauma patients and trauma outcomes research in Korea. J Acute Care Surg 2016;6:11-17.

21. Kim SY, So BH, Kim HM, Jeong WJ, Cha KM, Choi SP. Evaluation the usefulness of individual factors for determining the severity and predicting prognosis of trauma victims. J Trauma Inj 2015;28:134-43.

22. Fullerton JN, Price CL, Silvey NE, Brace SJ, Perkins GD. Is the modified Early Warning Score (MEWS) superior to clinician judgement in detecting critical illness in the pre-hospital environment? Resuscitation 2012;83:557-62. 